\title{
Marine Based Biomaterials in Dental Regeneration
}

\author{
Dr. Mekha Grace Varghese ${ }^{1}$, Dr. Thomas George V. ${ }^{2}$, Dr. Nebu George Thomas ${ }^{3}$, Dr. Alenya Mary Pyas ${ }^{4}$, \\ Dr. Arimboor Maymol Francis ${ }^{5}$ \\ ${ }^{1}$ Postgraduate Student, Department of Periodontics, ${ }^{2}$ Professor \& Head of Department, Department of Periodontics, \\ ${ }^{3}$ Professor, Department of Periodontics, ${ }^{4}$ Postgraduate Student, Department of Periodontics, ${ }^{5}$ Postgraduate Student, \\ Department of Prosthodontics
}

Pushpagiri College of Dental Sciences, Perumthuruthy P.O, Thiruvalla, Kerala, India

Corresponding Author: Dr. Mekha Grace Varghese; mekhagrace@gmail.com

Received 12 September 2020;

Accepted 30 September 2020;

Published 02 October 2020

\begin{abstract}
The novel approach of tissue engineering aims at regenerating the functional alveolar or periodontal tissues through a series of key events that is modulated by the use of scaffolds, cells and signalling molecules. Many synthetic and natural polymers have been used as tissue engineering constructs so far with varying results in regeneration. Developing a biomaterial to replace the damaged tissue is of paramount importance for effective regeneration. Due to its rich biodiversity, marine environment yields structures with immense potential for biomedical application. These bio molecules offer many applications in cartilage and bone tissue engineering, dental tissue regeneration, wound healing and local drug delivery system. These substances are usually nontoxic, bio compatible and well tolerated by the body, which boost their efficacy for tissue engineering application. In this article, we are trying to brief the various marine based biomaterials used in dental regeneration, their possible sources and clinical applications.
\end{abstract}

Keywords: Marine based, Bone grafts, Scaffolds, Chitosan, Collagen

\section{Introduction}

The attempts to reconstruct and augment the alveolar bone still remains an elusive goal for the maxillofacial and periodontal surgeons. Many patients can have significant amount of alveolar bone loss from physiological or pathological processes that includes aging, periodontal disease, osteoporosis, traumatic injury and neoplasms ${ }^{[1]}$. Moreover, in the last few decades, there is an increase in the life expectancy with a constant increase in number of patients seeking replacement of lost teeth with modalities like implants. This warrants the need to maintain sufficient vertical and horizontal alveolar ridge in adult population. The regenerative therapies in all these cases aims to increase the patients' bone mass. To date, the gold standard method adapted for alveolar bone regeneration is auto transplantation from patient's extra-oral or intra-oral donor sites. The method carries limitations like donor site morbidity, inadequate blood supply of graft tissue and limited availability. Alternative sources include the use of allografts and xenografts which also carries the possibility of immune rejection and virus transmission from donor to host. The widely used strategy of synthetic alloplasts are also discouraged due to their limited use in stress bearing situations ${ }^{[2]}$. So there is always the need for novel biomimetic biomaterials that can solve the current issues.

The regenerative surgical procedures widely applied for alveolar bone regeneration are mainly occlusive barrier membranes, bone grafts, growth factors and cell based therapies. Tissue engineering approaches using scaffolds alone or along with the critical elements like growth factors and cells can address the challenges encountered in bone regeneration ${ }^{[3]}$. The use of porous scaffolds provide a structural support for the cells to migrate, grow and differentiate leading to new bone formation. Many synthetic and natural polymers have been tried for fabrication of cell delivery systems and three dimensional scaffolds. Synthetic materials offer the advantage of more precisely controlled physico-chemical properties but they exhibit cell toxicity from the acidic by products of degradation. Conversely, natural polymers have inherent biologic cues and exhibits excellent biocompatibility and biodegradability ${ }^{[4]}$. The development of biodegradable biomaterials that can support the regrowth of tissues of periodontium is therefore gaining importance lately.

Thus there is a requirement of continuous identification of natural biomaterials to be used as bone grafts or scaffolds in tissue engineering fields, which led to the discovery of the marine ecosystem. The marine environment offers infinite diversity of biomaterials with broad spectrum of biological activities namely, antiviral, antibacterial, antifungal, antiprotozoal, anthelmintic, antiinflammatory, immunosuppressive, neuroprotective and non-toxic functions ${ }^{[5]}$. They offer excellent osteoconductivity and supports cell adhesion, proliferation and differentiation making them an attractive option in the regenerative scenario. Additionally, their 
cost effectiveness make them easily available to all strata of the society.

This review intends to describe the common marine based biomaterials used in dental field for the purpose of regeneration of periodontium as well as alveolar bone with emphasis on their origin and source, synthesis protocols as well as clinical application.

\section{Sources of Marine Biomaterials}

The marine environment is a remarkable source of natural compounds. With the help of continuous researches in the field of marine bio environment, an array of marine natural products has been established and they form dominant components of pharmaceuticals and tissue engineered constructs. Thus they emerge as valuable agents for periodontal regeneration and for alveolar ridge preservation.

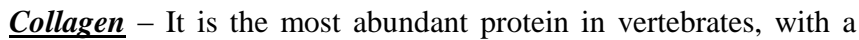
typical triple helical structure, making about $30 \%$ of the total protein mass. They play important role as a scaffolding material aiding in cell attachment, migration, proliferation and differentiation. Collagen can be extracted from numerous animal sources but the commonly used ones are bovine and porcine skin and tendons. They carry the risk of transmission of diseases like bovine spongiform encephalopathy and foot and mouth diseases. The purification process is also elaborate and expensive ${ }^{[6]}$.

Hence marine collagen is perceived as a tissue engineered substitute with the advantage of unique biological properties that includes water solubility, safety, biocompatibility, high biodegradability, low immunogenicity and easy extractability. It has better chemical and physical durability, abundant availability and is also free of the risks of transmission of viral animal diseases and pathogens ${ }^{[7]}$. Fish by-products such as skin, scale and bone are abundant sources of marine collagen ${ }^{[8]}$. The enormous amount of fish wastes discarded in the form of skins, bones, fins, heads, guts and scales can be converted into useful products of high significance and economic value ${ }^{[9]}$. Other important sources are corals, sponges, salmon, jellyfish, echinoderms and coralline algae. Of these, the collagen from marine sponges have properties like soft yet strong, highly absorbent, elastic and resistant to bacterial attack which make them suitable for scaffold fabrication ${ }^{[10]}$.

Chitin and chitosan - Chitin is one of the most abundant polysaccharides in nature. It is a long chain polymer of $\mathrm{N}$ - acetyl glucosamine and is the primary component of fungal cell walls, exoskeletons of arthropods and scales of fishes. Chitosan is a cationic polymer produced commercially through partial or full alkaline deacetylation of chitin ${ }^{[11]}$. Even though chitin is widely distributed throughout nature, marine crustaceans such as crabs, shrimp, lobsters, squid and krill fish are the convenient sources of chitin for commercial production. It is an easily available by product of the shell fish processing industry. A large proportion of chitin were found in aquatic and mollusca species belonging to phyla such as cnidaria, entoprocta, phoronida, ectoprocta, brachiopoda, bryozoan and porifera. Chitin was also detected in fungi, algae, coralline algae, green algae and protozoa ${ }^{[12]}$. It can be processed into different forms like powders, sponges, sheets, membranes and injectable medium ${ }^{[13]}$. They have got superior biodegradability, biocompatibility, low cytotoxicity and immunogenicity with critical roles in wound healing, hemostasis and immune enhancement. Their excellent antimicrobial properties enhance their use in tissue engineering applications ${ }^{[5]}$.

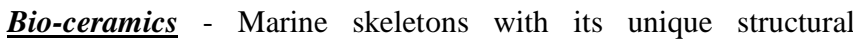
networks can function as templates for growth of human tissues. Corals, sponges, molluscs shells, cuttle fish, echinoderm spines and fish bones are the important sources of bio-ceramics ${ }^{[14]}$. A wide range of biomaterials derived from sea corals, sea shells, echinoderms, nacre, mediterranean mussel and cuttle fish can be used as raw material for synthesis of calcium phosphate compounds. Coral skeletons and converted coralline calcium phosphates are excellent to be used as scaffolds. The unique three dimensional structure enhances growth and differentiation of stem cells into bone cells. Calcium carbonates and phosphates such as hydroxyapatite (HA) have similarities with the mineral constituents of bones. The coral skeletal carbonate is unique because of their architectural properties like porosity, pore size and pore interconnectivity which are important in bone tissue regeneration $[15]$.

A serious disadvantage of marine-derived calcium carbonate skeletons are its fast dissolution rate and poor structural stability. These limitations affect their prolonged use in load bearing applications and hence they are converted more stable calcium phosphate structures like hydroxyapatites and tricalcium phosphate ${ }^{[16]}$. In this process, the carbonate component of the coral is exchanged for phosphate and its derivatives using high temperatures of 200- 260 degree centigrade for a period of 24-48 hours. The method was developed in 1974 by Roy and Linnehan [17] and is known as the hydrothermal exchange conversion strategy. Hydroxyapatite is one such important derivative obtained from biological source like fish bone, by the methods like calcination, hydrolysis etc. The bioactivity and stability exhibited makes it a unique material for bone tissue engineering. The excellent bone binding property by forming strong chemical bonds with surrounding bone is an important aspect of hydroxyapatite.

\section{Methods of Scaffold Fabrication from Marine Sources}

\section{Solvent Casting and Particle Leaching}

It is the conventional technique of scaffold fabrication where water soluble salt particles of a certain size are uniformly distributed and added to a solution of biodegradable polymer and an organic solvent. The solvent will then evaporates leaving a matrix containing salt particles which is then submerged in water to leach away the salt. The resultant obtained is a structure with high porosity. Scaffolds developed by this method have a porosity between $50 \%$ and $90 \%{ }^{[18]}$. Advantages include simplicity, versatility and ability to control pore size and geometry, but there is difficulty to accurately design pore interconnectivity ${ }^{[19]}$.

This technique is mainly used for fabrication of marine collagen and chitosan based scaffolds. Salt crystals are used as porogens and put into a mold along with chitosan. The small spaces left between the salt crystals are occupied by chitosan. The mold is then heated to melt the chitosan powder, in an oven which is later chilled. The chilled chitosan/ salt mixture is then separated from the mold and the salt is washed away by water or alcohol, which creates a porous architecture ${ }^{[20]}$.

\section{Freeze-Drying/ Lyophilisation}

This technique is commonly used to process the heat sensitive biological agents. In this technique, the polymer is dissolved in an appropriate organic solvent and water. After dissolution, the polymer solution is cooled under freezing point to form a solid solvent which is then subjected to sublimation leaving behind a solid scaffold with numerous interconnected pores. The principle is 
that when the solution is cooled to freezing point, solutes can be separated in the ice phase resulting in a small porous structure where there is matter surrounding the ice. The subsequent drying leaves behind a porous scaffold with porosity values upto $90 \%$. Advantage is that the pore size can be customized by changing the freezing method. Moreover, the use water and ice crystals instead of an organic solvent reduces cytotoxicity. Major limitation is the difficulty to ensure structural stability and mechanical properties of the constructs.

Collagen harvested from the jellyfish can be freeze dried to obtain scaffolds ${ }^{[21]}$. Biphasic scaffolds, thus fabricated, from salmon collagen, alginate and jellyfish collagen supported the differentiation of chondrogenic and osteogenic cells from bone marrow mesenchymal cells ${ }^{[22]}$.

Freeze-drying and lyophilizing of chitosan solution is a common method to generate chitosan scaffolds. Ice crystals occupy spaces in the frozen chitosan solution which are then emptied during the sublimation, leading to the formation of pores. However, a precise control of temperature is required for formation of good pore structure ${ }^{[23]}$.

\section{Electrospinning}

The technique of making fibres from a solution by using electric current is electrospinning. This technique helps in developing nanofibrous scaffolds in tissue engineering. A standard electrospinning system consists of four main components: a spinner with a syringe pump, a metallic needle, a high-voltage power supply and a grounded collector. In this system, the liquid is charged under high voltage. When the strength of the electric field exceeds the surface tension of the droplet, a liquid jet is produced that is then extended and whipped continuously. The jet is solidified to form into a nonwoven nano/micro sized fibrous membrane ${ }^{[24]}$.

Marine collagen and hydroxyapatite based composite nanofibrous scaffolds were fabricated using this technique from fish scale and skin, which helps in guided bone regeneration ${ }^{[25]}$. Tilapia skin derived collagen along with bioglass were also fabricated using electrospinning and is used as a wound dressing to promote wound healing ${ }^{[26]}$. In addition, fibrous chitosan scaffolds are also formed by electrospinning. When an electric field is applied, there is elongation of the chitosan drop and formation of long fibres with varying diameters ${ }^{[27]}$.

\section{Three-Dimensional Printing (3DP)}

3DP is a new fabrication method for tissue engineering utilized for precise control of scaffold structure at the micron level. It has the ability to control bulk geometry and internal structure of tissue scaffolds. It is a rapid prototyping method, where a functional prototype is created directly from the computer models ${ }^{[28]}$. The technique allows printing of multi layered scaffolds seeded with different cell types in each layer thus forming tissues that mimic the original structure. It is widely used for processing of natural polymers like chitosan or chitosan composites with hydroxyapatite. Since this method uses low temperature, the incorporation of cells and bioactive molecules is also possible.

Bioprinting is a $3 \mathrm{D}$ printing technique, defined as "using material transfer processes for developing a biological pattern and assembly of relevant materials, cells, molecules, tissues, and biodegradable biomaterials with a prescribed structure to achieve some biological functions" ${ }^{[29]}$. This systems allows the direct printing of biomaterials on three-dimensional scaffolds for transplantation with/ without seeded cells.

Marine derived biomaterials used as bioinks in 3D bioprinting are alginate, chitosan, carrageenan, collagen, and gelatin. Compared to mammalian resources, marine natural resources do not carry risks of transmissible diseases and are unaffected by religious restrictions. They also enable high volume production at a low cost. Alginate has fast gelation process and rheological properties. The other desirable features like biocompatibility, biodegradability and ease of manipulation make them good candidates for tissue regeneration and drug delivery. Gelatin from marine resources, such as f-gelatin, provides a promising alternative with the advantages of low gelling temperature and low melting point. Hence it is an attractive option in tissue engineering but it carries the limitation of rapid degradation which makes them less suitable for forming stable hydrogels ${ }^{[30]}$.

Chitosan has the feasibility and printability as a bio printing solution. Chemical modification of amino groups of chitosan backbone alters there mechanical and biological properties. But the thermo-mechanical processing is not suitable for chitosan because it degrades before melting. The processing temperature must be controlled to avoid degradation. Hydroxyapatite, when in physiological solution, shows marginal matrix stability. Therefore they should be esterified and photo cross-linked prior to 3D bioprinting. Collagen is excellent to be used as a bio ink due to their good water solubility, low melting point and low viscosity ${ }^{[31]}$.

By bioprinting, hybridization of natural and synthetic materials is possible thus combining the advantages of both like mechanical strength and controlled degradation. Thus it's a promising approach to create novel tissue engineered scaffolds which are patient specific ${ }^{[32]}$.

Robocasting, another variant of $3 \mathrm{D}$ printing, is a recent additive manufacturing technique which enables the production of scaffolds with precise control of internal scaffold architecture without the need for subsequent machining. Here, the scaffolds are built by robotic deposition of a continuous filament capable of fully supporting their own weight. The deposition process is conducted in a non-wetting oil bath to prevent non-uniform drying and a layer by layer assembly takes place through a nozzle ${ }^{[33]}$.

\section{Clinical Application}

\section{Dental tissue engineering}

Marine biomaterials like collagen, alginates and chitosan have been employed for a wide variety of applications in dentistry. Collagen isolated from fresh water fish like tilapia had been used in dentin and pulp regeneration ${ }^{[34]}$. They can also enhance viability of human periodontal ligament stem cells and up regulated the expression of osteogenic markers thus aiding in alveolar bone regeneration ${ }^{[35]}$.

Fish derived collagen can be used as membranes, local delivery agents and haemostatic agents in dentistry ${ }^{[36]}$. The marine collagen derived membranes can be used as barrier membranes for guided tissue regeneration and guided bone regeneration aiding in periodontal regeneration and implant site development. Collagen fibres are also used as a carrier for chlorhexidene and tetracycline aiding in local drug delivery in periodontal diseases. As the collagen undergoes resorption, the drug is released from the matrix in a controlled manner. The sponge like structure of collagen makes it a highly absorbent material. Hence, they can be used to control bleeding at surgical sites by creating an artificial clot ${ }^{[37]}$.

Another principal agent for dental application is chitosan due to its excellent biocompatibility, bioactivity and antimicrobial properties. Chitosan hydrogels are widely used as bone defect fillers when treating chronic periodontitis. The antimicrobial 
activity of chitosan on oral biofilms as well as the ability to reduce concentrations of Streptococcus mutans makes them a good candidate to be incorporated into tooth pastes and mouth washes.

The regenerative strategies using scaffolds are also used targeting regeneration of pulp and dentin ${ }^{[38]}$. The scaffolds helps to deliver active molecules and competent cells to the endodontic compartment. Chitosan based scaffolds are favourable for both pulp and dentin regeneration ${ }^{[39]}$. When incorporated with betatricalcium phosphate, they increased expression of mineralization markers, such as osteopontin and alkaline phosphatase. They promoted the proliferation, migration and odontoblastic differentiation of dental pulp stem cells and mesenchymal stem cells both in vitro and in vivo ${ }^{[40]}$. They also promoted dentin formation by human periodontal ligament cells ${ }^{[41]}$.

\section{Bone tissue engineering}

Marine collagen is used as a biomaterial in bone tissue engineering because of their substantial osteogenic activity. In a study by Yamada et al, in 2013, marine collagen extracted from the scales of tilapia induced multidirectional differentiation in the primary rat bone marrow mesenchymal stem cells. They promoted cell viability and significantly up regulated the expression of osteogenic markers. There was increased cell proliferation, alkaline phosphatase activity and mineralization in osteoblast cell cultures for bone tissue engineering ${ }^{[42]}$. Further studies have confirmed that matrix mineralization and collagen deposition were also accelerated. On human mesenchymal stem cells, marine collagen increased adherence, accelerated osteoblast differentiation and up regulated the osteoblastic markers, facilitating osteoblast differentiation. They stimulated osteogenic potential of osteoblasts and inhibited proliferation of osteoclasts thereby preventing osteoporosis $^{[43,44]}$.

Calcium phosphate compounds can be easily obtained from coralline material and has the unique properties of porosity, architecture and pore interconnectivity. They can produce firm attachment with the host tissue and permits circulation of body fluids through them. The biocompatible and osteoconductive nature also make them an ideal candidate for bone tissue engineering ${ }^{[17]}$.

Incorporation of hydroxyapatite with chitin is another promising agent for bone substitution due to their non-cytotoxic nature and biodegradability ${ }^{[45]}$. Bio composite scaffolds, made of chitosan matrix incorporated with chitin nano-crystals, chitosan cross-linked with collagen and cellulose nano-fibre reinforced chitosan are ideal for bone tissue engineering due to their ability to promote osteoblast cell adhesion and proliferation. They also carry optimum degradation and mechanical properties required for bone scaffolds. These bio composites can also promote attachment, migration and proliferation of Schwann cells helping in nerve tissue regeneration.

Chitosan is widely used for the purpose of bone regeneration since it can be easily processed and manufactured in a variety of forms including fibres, films, sponges and hydrogels thus helping to mimic the shape of the receiving tissue or a biomaterial tissue interface ${ }^{[46]}$. They are physiologically inert and relatively safe. Upon implantation or topical application, no allergic reactions occurred in the human body ${ }^{[47]}$. Because of their reduced mechanical properties, chitosan alone as a scaffold has limited use. But when mixed with other synthetic (polycaprolactone) or natural (alginate, collagen and silk fibroin) polymers or bioceramics (hydroxyapatite, beta tricalcium phosphate) or bioactive molecules (bone morphogenetic protein-2, vascular endothelial growth factor), they form excellent scaffolds for bone tissue regeneration. These bio-composite scaffolds are non-toxic, biocompatible, osteoconductive and osteogenic, thereby promoting cell proliferation and adhesion leading to new bone formation.

\section{Cartilage tissue engineering}

Cartilages have very limited ability to regenerate due to their avascular nature. Collagen from skin of deep water ocean fishes can enhance the chondrogenic differentiation of adipose derived stromal cells. They also promote chondrogenesis in human mesenchymal stem cells with up regulation of chondrogenic markers and down regulation of osteogenic markers ${ }^{[48]}$.

Coralline materials form novel scaffolds for cartilage tissue regeneration. Due to their superior properties of biocompatibility, biodegradability, osteogenic capacity and stimulatory effect on cellular invasion, adherence and proliferation, they can be effectively used for cartilage regeneration ${ }^{[49]}$.

Chitosan is also widely used in cartilage regeneration. Their structure resembles the glycosaminoglycans found in articular cartilages ${ }^{[50]}$. Chitosan along with marine polysaccharides like hyaluronin can improve chondrocyte adhesion and proliferation. They can also be blended with other materials like silk fibroin and gelatin which functions as excellent carriers of stem cells to repair cartilage defects ${ }^{[51]}$.

\section{Wound healing}

Collagen from marine sources had stimulating effects on fibroblasts proliferation, collagen synthesis and re-epithelialization thereby assisting in wound contraction and dermal reconstitution. They also up regulated expression of epidermal growth factor and fibroblast growth factor ${ }^{[52]}$. Hence they are valuable agents for scaffold fabrication. The dual properties of wound healing and antimicrobial activity makes them an optimal dressings suitable for wound management. Alginate with its property for skeletal muscle regeneration is an ideal agent for grafting in deep thickness wound $[53]$.

Chitosan hold many desirable qualities of non-toxicity, biodegradability, biocompatibility and antimicrobial property that helps in wound healing. They have effects on hemostasis as well as immune enhancement. When blended with other marine derived materials, they can be used for fabrication of hydrogel dressings that increase granulation tissue formation and vascularization ${ }^{[54]}$. Chitosan structure has a positively charged polysaccharide molecule with amino groups on its back bone. These positively charged molecules has electrostatic interaction with negatively charged molecules on the surface of red blood cells. This interaction increases blood viscosity, activates platelet adhesion and aggregation and enhances platelet transportation to the vessel wall bringing about hemostasis. The hemostatic property is controlled by the number of positive charged amino groups on chitosan ${ }^{[5,56]}$.

The cationic nature of chitosan is also responsible for its antimicrobial activity on a wide variety of micro-organism including bacteria, yeast, fungi and algae. Chitin films and alginate films from marine sources, with its flexibility, transparency, cyto compatibility and adherence property, also forms ideal wound dressing materials.

\section{Conclusion}

The last decade has witnessed the evolution of biomimetic marine derived materials and their wide use in tissue regeneration approaches. There is always a need for better tissue engineering scaffolds that is more bio-responsive thereby guiding the natural processes of tissue regeneration. The concept of 'intelligent scaffolds' is emerging that will meet this biological challenge. 
Biomaterials has the ability to constantly adapt to dynamically changing environmental conditions by altering their composition, growth and function. They also show a self-repairing ability when adaptation fails. Marine structures have unique designs, chemistry, adaptability and functionality which makes them highly demanded in the field of regenerative medicine and dentistry. Marine biotechnology allows exploration of the marine body abundant with a wide variety of organisms some of which are already being used as tissue engineering substitutes. Future studies are needed that warrants the identification of biomaterials with immense potential for tissue engineering application.

\section{Source(s) of support}

Nil

\section{Presentation at a meeting}

Nil

\section{Conflicting Interest}

Nil

\section{References}

[1] Sowmya, S, Mony U, Jayachandran P, et al. Tri-Layered Nano composite Hydrogel Scaffold for the Concurrent Regeneration of Cementum, Periodontal Ligament, and Alveolar Bone. Adv. Healthc. Mater. 2017; 6:1601251.

[2] Funda G, Taschieri S, Bruno GA, et al. Nano technology scaffolds for alveolar bone regeneration. Materials (Basel). 2020; 13: 201.

[3] Pilipchuk SP, Plonka AB, et al. Tissue engineering for bone regeneration and osseointegration in the oral cavity. Dent Mater 2015; 31: 317-338.

[4] Chocholata P, Kulda V, Babuska V. Fabrication of scaffolds for bone tissue regeneration. Materials (Basel). 2019; 12: 568.

[5] Chandika P, Ko SC, Jung WK. Marine derived biological macromolecule- based biomaterials for wound healing and skin tissue regeneration. Int J Biol Macromol. 2015; 77: 24-35.

[6] Colchester A.C, Colchester N.T. The origin of bovine spongiform encephalopathy: The human prion disease hypothesis. Lancet. 2005; 366: 856-861.

[7] Lim YS, Ok YJ, Hwang SY, Kwak JY, Yoon S. Marine Collagen as A Promising Biomaterial for Biomedical Applications. Mar Drugs. 2019; 17:467.

[8] Cho J K, Jin Y G, Rha S J, Kim S J. Hwang J H. Biochemical characteristics of four marine fish skins in Korea. Food Chem. 2014; 159: 200-207.

[9] Caruso G. Fishery Wastes and By-products: A Resource to Be Valorised. J. Fish. Sci. 2015; 9: 80-83.

[10] Ben-Nissan B, Choi AH, Green DW. Marine derived biomaterials for bone regeneration and tissue engineering: Learning from nature. In: Choi A, BenNissan B (eds) Marine derived biomaterials for tissue engineering applications. Springer series in Biomaterial Science and Engineering, Vol 14. Springer, Singapore.

[11] Andres Y, Giraud L, Gerente C, Le Cloirec P. Antibacterial effects of chitosan powder: mechanisms of action. Environ Technol. 2007; 28:1357-1363.
[12] Kaur S, Dhilol GS. The versatile biopolymer Chitosan: potential sources, evaluation of extraction methods and applications. Crit.Rav.Microbiology. 2014; 40: 155-175.

[13] Aguilar A, Zein N, Harmouch E, et al. Application of Chitosan in Bone and Dental Engineering. Molecules. 2019; 24:3009.

[14] Silva T H, Alves A, et al. Materials of marine origin: a review on polymers and ceramics of biomedical interest. International Materials Reviews. 2012; 57: 276-306.

[15] Laine J, Labady M, Albornoz A, Yunes S. Porosities and pore sizes in coralline calcium carbonate. Mater. Charact. 2008; 59: 1522-1525.

[16] Chou J, Ben-Nissan B, Choi AH et al. Conversion of coral sand to calcium phosphate for biomedical application. J Aust Ceram Soc. 2007; 43:44-48.

[17] Roy DM, Linnehan SK. Hydroxyapatite formed from coral skeletal carbonate by hydrothermal exchange. Nature. 1974; 247: 220-222.

[18] Li Z, Xie M B, Li Y, Ma Y, Li J S, Dai F Y. Recent progress in tissue engineering and regenerative medicine. Journal of Biomaterials and Tissue Engineering. 2016; 6: 755-766.

[19] Moore M J, Jabbari E, Ritman E L, Lu L C, Currier B L, Windebank A J, Yaszemski M J. Quantitative analysis of interconnectivity of porous biodegradable scaffolds with micro-computed tomography. J. Biomed. Mater. Res. Part A. 2004; 71:258-267.

[20] Lim J I, Lee Y K, Shin J S, Lim K J. Preparation of interconnected porous chitosan scaffolds by sodium acetate particulate leaching. J. Biomater. Sci. Polym. Ed. 2011; 22: 1319-1329.

[21] Pustlauk W, Paul B, Gelinsky M, Bernhardt A. Jellyfish collagen and alginate: Combined marine materials for superior chondrogenesis of hMSC. Mater. Sci. Eng. C. 201; 64: 190-198.

[22] Bernhardt A, Paul B, Gelinsky M. Biphasic Scaffolds from Marine Collagens for Regeneration of Osteochondral Defects. Mar. Drugs. 2018; 16: 91.

[23] Keller L, Regiel-Futyra A, Gimeno M, et al. Chitosanbased nanocomposites for the repair of bone defects. Nanomedicine 2017; 13: 2231-2240.

[24] Li Z, Xie M B, Li Y, Ma Y, Li J S, Dai F Y. Recent progress in tissue engineering and regenerative medicine. Journal of Biomaterials and Tissue Engineering. 2016; 6: 755-766.

[25] Jin S, Sun F, Zou Q et al. Fish Collagen and Hydroxyapatite Reinforced Poly (lactide-co-glycolide) Fibrous Membrane for Guided Bone Regeneration. Biomacromolecules. 2019; 20: 2058-2067.

[26] Zhou T, Sui B, Mo X, Sun J. Multifunctional and biomimetic fish collagen/bioactive glass nano fibers: Fabrication, antibacterial activity and inducing skin regeneration in vitro and in vivo. Int. J. Nanomed. 2017; 12: 3495-3507.

[27] Dahlin R L, Kasper F K, Mikos A G. Polymeric nanofibers in tissue engineering. Tissue Eng. Part. B Rev. 2011; 17: 349-364.

[28] Seitz H, Rieder W, Irsen S, Leukers B, Tille C. Three dimensional printing of porous ceramic scaffolds for bone tissue engineering. J. Biomed. Mater. Res. Part B Appl. Biomater. 2005; 74:782-788.

[29] Murphy S V, Atala A. 3D bioprinting of tissues and organs. Nat Biotech. 2014; 32: 773-785. 
[30] Zhang X, Kim G, et al. Marine Biomaterial-Based Bioinks for Generating 3D Printed Tissue Constructs. Marine Drugs. 2019; 16: 484.

[31] Zhang Y, Zhou D, Chen J, Zhang X, Li X, Zhao W, Xu T. Biomaterials Based on Marine Resources for 3D Bioprinting Applications Mar Drugs. 2019; 17: 555.

[32] Zhang L, Yang G, Johnson B N, Jia X. Threedimensional (3D) printed scaffold and material selection for bone repair. Acta Biomaterialia. 2019; 84: 16-33.

[33] Neto AS, Ferreira MF. Synthetic and marine derived porous scaffolds for bone tissue engineering. Materials (Basel). 2018; 11:1702.

[34] Tang J, Saito T. Biocompatibility of novel type 1 collagen purified from tilapia fish scale: An in vitro comparative study. Bio Med Res. Int. 2015; 2015: 139476.

[35] Liu C, Sun J. Hydrolysed tilapia fish collagen induces osteogenic differentiation of human periodontal ligament cells. Biomed. Mater. 2015; 10: 065020.

[36] Lim YS, Ok YJ, Hwang SY, Kwak JY, Yoon S. Marine collagen as a promising biomaterial for biomedical applications. Mar Drugs. 2019; 17: 467.

[37] Mahesh L, Kurtzman G M, Shukla S. Regeneration in Periodontics: Collagen - A review of its properties and applications in dentistry. Compend Contin Educ Dent. 2015; 36: 358-363.

[38] Keller L, et al. Active Nanomaterials to Meet the Challenge of Dental Pulp Regeneration. Mater. (Basel) 2015; 8: 7461-7471.

[39] Zein N, Harmouch E, et al Polymer-Based Instructive Scaffolds for Endodontic Regeneration. Mater. (Basel) 2019; 12: 2347.

[40] Ducret M, Montembault A, et al. Design and characterization of a chitosan-enriched fibrin hydrogel for human dental pulp regeneration. Dent. Mater. 2019; 35: 523-533.

[41] Liao F, Chen Y, Li Z, Wang Y, Shi B, Gong Z, Cheng X. A novel bioactive three-dimensional beta-tricalcium phosphate/chitosan scaffold for periodontal tissue engineering. J. Mater. Sci. Mater. Med.2010; 21: 489496.

[42] Yamada S, Yoshizawa Y, Kawakubo A, Ikeda T, Yanagiguchi K, Hayashi Y. Early gene and protein expression associated with osteoblast differentiation in response to fish collagen peptides powder. Dent. Mater. J. 2013; 32: 233-240.

[43] Matsumoto R, Uemura T, Xu Z, Yamaguchi I, Ikoma T, Tanaka J. Rapid oriented fibril formation of fish scale collagen facilitates early osteoblastic differentiation of human mesenchymal stem cells. J. Biomed. Mater. Res. A 2015; 103: 2531-2539.

[44] $\mathrm{Hu} \mathrm{CH}$, Yao CH, et al. Effects of different concentrations of collagenous peptide from fish scales on osteoblast proliferation and osteoclast resorption. Chin. J. Physiol. 2016; 59: 191-201.

[45] Ge Z, Baguenard S, Lim LY et al. Hydroxyapatite-chitin materials as potential tissue engineered bone substitutes. Biomaterials. 2004; 25:1049-1058.

[46] Kim I Y, Seo S J, Moon H S et al. Chitosan and its derivatives for tissue engineering applications" Biotechnology advances. 2008; 26: 1-21.

[47] Azuma K, Osaki T, Minami S, Okamoto Y. Anticancer and anti-inflammatory properties of chitin and chitosan oligosaccharides. J. Funct. Biomater. 2015; 6: 33-49.

[48] Hsu H, Uemura T, Yamaguchi I, Ikoma T, Tanaka J. Chondrogenic differentiation of human mesenchymal stem cells on fish scale collagen. J. Biosci. Bioeng. 2016; 122: $219-225$.

[49] Demers C, Hamdy CR, Corsi K et al. Natural coral exoskeleton as a bone graft substitute: a review. Biomed Mater Eng. 2002; 12:15-35.

[50] NgeTT, Nogi $\mathrm{M}$, Yano $\mathrm{H}$ et al. Microstructure and mechanical properties of bacterial cellulose/ chitosan porous scaffold. Cellulose. 2010; 17:349-363.

[51] Deng J, She R, Huang W et al. A silk fibroin/chitosan scaffold in combination with bone marrow-derived mesenchymal stem cells to repair cartilage defects in the rabbit knee. J Mater Sci Mater Med. 2013; 24:20372046.

[52] Chen J, Gao K, Liu S, Wang S, Elango J, Bao B, Dong J, Liu N, Wu W. Fish Collagen Surgical Compress Repairing Characteristics on Wound Healing Process In Vivo. Mar. Drugs 2019; 17: 33.

[53] Rabbany SY, Pastore J, Yamamoto $M$ et al. Continuous delivery of stromal cell-derivedfactor- 1 from alginate scaffolds accelerates wound healing. Cell Transplant. 2010; 19: 399-408

[54] Murakami K, Aoki H, Nakamura S et al. Hydrogel blends of chitin/chitosan, fucoidan and alginate as healing-impaired wound dressings. Biomaterials. 2010; 31:83-90.

[55] Hu Z, Zhang DY, Lu ST, Li PW, Li SD. Chitosan-Based Composite Materials for Prospective Hemostatic Applications. Mar. Drugs. 2018; 16: 273.

[56] Chou TC, Fu E, Wu CJ, Yeh JH. Chitosan enhances platelet adhesion and aggregation. Biochem. Biophys. Res. Commun. 2003; 302: 480-483. 